\title{
PRIME DIVISORS OF SOME SHIFTED PRODUCTS
}

\author{
ERIC LEVIEIL, FLORIAN LUCA, AND IGOR E. SHPARLINSKI
}

Received 12 May 2005 and in revised form 31 August 2005

We study prime divisors of various sequences of positive integers $A(n)+1, n=1, \ldots, N$, such that the ratios $a(n)=A(n) / A(n-1)$ have some number-theoretic or combinatorial meaning. In the case $a(n)=n$, we obviously have $A(n)=n$ !, for which several new results about prime divisors of $n !+1$ have recently been obtained.

\section{Introduction}

We denote by $P(m)$ the largest prime factor of a positive integer $m$.

Let $N \geq 1$ be sufficiently large. Assume that a sequence of positive rational numbers $a(i)$ for $i=1, \ldots, N$ is such that

$$
A(n)=\prod_{i=1}^{n} a(i)
$$

takes integer values for every $n=1, \ldots, N$. For several such sequences, we get lower bounds on the largest prime factor of the "shifted" products $P(A(n)+1)$.

More specifically, we consider

(i) products of consecutive values of an integer-valued polynomial $g(X) \in \mathbb{Q}[X]$ :

$$
G(n)=\prod_{i=1}^{n}|g(i)| ;
$$

(ii) products of consecutive values of an arithmetic function

$$
F(n)=\prod_{i=1}^{n} f(i),
$$

where $f(i)$ is one of the following functions: $\varphi(i)$ the Euler function, $\sigma(i)$ the sum of divisors function, $\tau(i)$ the number of divisors function, $\Omega(i)$ and $\omega(i)$ the number of prime factors function (counted with and without multiplicities, resp.); 
(iii) products of middle binomial coefficients

$$
M(n)=\prod_{i=1}^{n}\left(\begin{array}{c}
2 i \\
i
\end{array}\right)
$$

(iv) $q$-factorials

$$
F_{q}(n)=n !_{q}=\prod_{i=1}^{n}\left(q^{i}-1\right),
$$

where $q \geq 2$ is a fixed integer;

or slight variations of those.

Our approach generally follows that of $[3,4,6]$, where similar questions are considered for $n$ !. However, treatment of each of the above sequences also requires some specific ingredients.

Throughout the paper, we use the Vinogradov symbols $\gg, \ll$, and $\asymp$, as well as the Landau symbols $O$ and $o$ with their regular meanings. We recall that $U \ll V$ and $U=$ $O(V)$ are both equivalent to the inequality $|U| \leq c V$ with some constant $c>0$. Furthermore, we say that two functions $U$ and $V$ are "equivalent" and write $U \sim V$ if $U=$ $(1+o(1)) V$.

For $z>0$, we use $\log z$ to denote the natural logarithm of $z$. For a positive integer $k \geq 2$, we write $\log _{k} z$ for the composition of $\log$ with itself $k$ times evaluated at $z$. We use the letter $p$ to denote a prime number, and $\pi(z)$ to denote the number of prime numbers $p \leq z$.

\section{General framework}

Here, we present our results in the most generic form even if this is somewhat technically cluttered. Our bounds are based on assumed growth conditions and divisibility properties of our sequences, which are readily available for many interesting concrete examples.

Throughout this paper, we assume that $a(n)>1$ holds for all $n=1,2, \ldots$, and also that

$$
\log _{2} A(n) \geq(1+o(1)) \log n \quad \text { as } n \longrightarrow \infty .
$$

2.1. Notation and preparations. Let $d \geq 1$ be fixed integer. Our goal is to prove that there exist at least $d$ positive integers $n \leq N$ such that $P(A(n)+1)$ is quite large.

For $n \leq N$, we write

$$
\begin{gathered}
\mathbf{b}(N)=\sum_{n=1}^{N} \log A(n), \\
\mathbf{W}(N)=\log \left(\prod_{n=1}^{N}(A(n)+1)\right) .
\end{gathered}
$$


We remark that

$$
\mathbf{W}(N)=\sum_{n=1}^{N} \log (A(n)+1) \geq \sum_{n=1}^{N} \sum_{i=1}^{n} \log a(i)=\mathbf{b}(N) .
$$

We need two functions $\vartheta(p)$ and $\eta(p)$ such that

$$
A(n)+1 \not \equiv 0 \quad(\bmod p), \text { for } n<\vartheta(p), n>\eta(p) .
$$

Obviously, we can always take $\vartheta(p)=1$ and $\eta(p)=N$, but in what follows we always assume that $\vartheta(p)$ is the smallest positive integer such that $p \mid A(\vartheta(p))+1$.

We note that if $p \mid A(n)+1$ for some $n \leq N$, then

$$
\vartheta(p) \leq n \leq \eta(p)
$$

We define

$$
\Delta(p)=\eta(p)-\vartheta(p)
$$

We now write $a(i)=u(i) / v(i)$ with coprime positive integers $u(i), v(i)$, set $h(i)=\max \{u(i)$, $v(i)\}$, and put

$$
\mathbf{h}(n)=\max _{1 \leq i \leq n} \log (h(i)) .
$$

Note that $h(i)$ is the naïve height of $a(i)$.

Let $\mathscr{P}$ be the set of all the prime factors of $\prod_{n=1}^{N}(A(n)+1)$. Thus,

$$
\prod_{n=1}^{N}(A(n)+1)=\prod_{p \in \mathscr{P}} p^{\alpha(p)}
$$

For a prime number $p \in \mathscr{P}$, we write

$$
s(p)=\max \left\{s: p^{s} \mid A(n)+1 \text { for some } n=1, \ldots, N\right\},
$$

and for $1 \leq s \leq s(p)$, we write

$$
t(p, s)=\#\left\{n: 1 \leq n \leq N, A(n) \equiv-1\left(\bmod p^{s}\right)\right\} .
$$

We often use the following combinatorial fact that

$$
\sum_{p \in \mathscr{P}} \alpha(p) \log p=\sum_{p \in \mathscr{P}} \sum_{s=1}^{s(p)} t(p, s) \log p .
$$

Lemma 2.1. The following bound holds:

$$
t(p, s) \leq 1+\frac{\mathbf{h}(N)}{s \log p-\log 2} \Delta(p) .
$$


3060 Prime divisors of some shifted products

Proof. We can suppose that $t(p, s) \geq 2$, otherwise there is nothing to prove. Using (2.5), we obtain that there must exist two integers $n$ and $m$ with

$$
\vartheta(p) \leq n<m \leq \eta(p), \quad m-n \leq \frac{\Delta(p)}{t(p, s)-1}
$$

such that $p^{s}$ divides both $A(n)+1$ and $A(m)+1$. Since

$$
A(m)+1=(A(n)+1) \prod_{i=n+1}^{m} a(i)-\left(\prod_{i=n+1}^{m} a(i)-1\right)
$$

we get that

$$
(A(m)+1) \prod_{i=n+1}^{m} v(i)=(A(n)+1) \prod_{i=n+1}^{m} u(i)-\left(\prod_{i=n+1}^{m} u(i)-\prod_{i=n+1}^{m} v(i)\right)
$$

an equation which when reduced modulo $p^{s}$ gives

$$
p^{s} \mid\left(\prod_{i=n+1}^{m} u(i)-\prod_{i=n+1}^{m} v(i)\right)
$$

The number appearing on the right-hand side of the above divisibility formula is nonzero (because $a(i)>1$ for all $i=n+1, \ldots, m$ ), therefore

$$
p^{s} \leq\left|\prod_{i=n+1}^{m} u(i)-\prod_{i=n+1}^{m} v(i)\right| \leq 2 \prod_{i=n+1}^{m} h(i)
$$

which gives

$$
s \log p \leq \log 2+\sum_{i=n}^{m} \log h(i) \leq \log 2+(m-n) \mathbf{h}(N),
$$

and finishes the proof.

We remark that we have a simple upper bound on $t(p, s)$, that is $\Delta(p)$. We choose to deal with a more general case, and we suppose that we have

$$
t(p, s) \leq D \Delta(p)^{1-\rho(p)}
$$

where $D$ is a constant and $0 \leq \rho(p) \leq \rho<1$ for some constant $\rho$.

For many sequences, we have the bound (2.19) with some specific values of $D$ and $\rho(p)$ (see Section 3 for some specific examples). 
Eric Levieil et al. 3061

We recall that

$$
\sum_{k=i}^{j} \frac{1}{k} \leq \frac{1}{i}+\int_{i}^{j} \frac{d t}{t}=\log \left(\frac{j}{i}\right)+1
$$

From now on, we assume that

$$
\log (\mathbf{h}(N)) \geq D+2 \text {. }
$$

We put

$$
\Psi(p)=\mathbf{h}(N) \Delta(p)\left(\log _{2} A(N)-\rho(p) \log \Delta(p)\right) .
$$

LEMmA 2.2. If the bound (2.19) holds, then

$$
\sum_{s=1}^{s(p)} t(p, s) \log p \leq \log (A(N)+1)+\Psi(p)\left(1-\frac{\log 2}{\mathbf{h}(N)}\right)^{-1},
$$

provided that $N$ is sufficiently large.

Proof. We put

$$
s_{1}(p)=\left\lfloor\frac{\mathbf{h}(N) \Delta(p)^{\rho(p)}}{\log p}\right\rfloor .
$$

We first assume that $s_{1}(p)<s(p)$. We then put $s_{2}(p)=s_{1}(p)+1$. Using the bound (2.19), we obtain the inequality

$$
\sum_{s=1}^{s_{1}(p)} t(p, s) \log p \leq D \mathbf{h}(N) \Delta(p)
$$

Using Lemma 2.1 and the trivial bound $s(p) \log p \leq \log (A(N)+1)$, we obtain

$$
\begin{aligned}
\sum_{s=s_{2}(p)}^{s(p)} t(p, s) \log p & \leq \sum_{s=s_{2}(p)}^{s(p)}\left(\log p+\frac{\mathbf{h}(N) \Delta(p)}{s(1-\log 2 / s \log p)}\right) \\
& \leq s(p) \log p+\mathbf{h}(N) \Delta(p)\left(1-\frac{\log 2}{s_{2}(p) \log p}\right)^{-1} \sum_{s=s_{2}(p)}^{s(p)} \frac{1}{s} \\
& \leq \log (A(N)+1)+\mathbf{h}(N) \Delta(p)\left(1-\frac{\log 2}{\mathbf{h}(N)}\right)^{-1}\left(\log \left(\frac{s(p)}{s_{2}(p)}\right)+1\right),
\end{aligned}
$$

and the proof in this case finishes by using

$$
\log \left(\frac{s(p)}{s_{1}(p)+1}\right)+1 \leq \log \left(\frac{\log (A(N)+1)}{\mathbf{h}(N) \Delta(p)^{\rho(p)}}\right)+1 \leq \log \left(\frac{\log A(N)}{\Delta(p)^{\rho(p)}}\right)-D
$$


3062 Prime divisors of some shifted products

and the inequality (2.25). In the last chain of inequalities above we used, besides the inequality (2.21), also the inequality

$$
\log (\log (A(N)+1)) \leq 1+\log (\log A(N)),
$$

which is equivalent to the fact that $A(N)^{e} \geq A(N)+1$, which in turn holds because $A(N) \geq 2$.

Assume now that $s_{1}(p) \geq s(p)$. Since $\Delta(p) \leq N$, recalling (2.1), we see that

$$
\log _{2} A(N)-\rho(p) \log \Delta(p) \geq(1-\rho+o(1)) \log N \text {. }
$$

Hence,

$$
\Psi(p) \geq \mathbf{h}(N) \Delta(p)(1-\rho+o(1)) \log N
$$

which together with the estimate (2.25) shows that the desired inequality holds if $N$ is large enough.

2.2. General bounds. We have the following theorem.

Theorem 2.3. Let $d$ be fixed and let $\Theta(n)$ be an increasing function such that the inequality $\vartheta(\Theta(n)) \leq n$ holds for all $n \geq 1$. If $N$ is sufficiently large and

$$
(\mathbf{b}(N-d)-\pi(\Theta(N)) \log (A(N)+1))\left(1-\frac{\log 2}{\mathbf{h}(N)}\right) \geq \sum_{p \leq \Theta(N)} \Psi(p),
$$

then there are at least $d$ positive integers $n \leq N$ with

$$
P(A(n)+1) \geq \Theta(n) .
$$

Proof. We suppose that there are only $e<d$ positive integers $n \leq N$ with $P(A(n)+1) \geq$ $\Theta(n)$. We have

$$
\mathbf{W}(N)=\sum_{p \in \mathscr{P}} \sum_{s=1}^{s(p)} t(p, s) \log p
$$

Since $a(n)>1$ holds for all positive integers $n$, we infer that if we eliminate from each side of the above identity the terms that come from the $e$ integers mentioned above, we obtain

$$
\mathbf{W}(N-e) \leq \sum_{p \leq \Theta(N)} \sum_{s=1}^{s(p)} t(p, s) \log p
$$

Using (2.3) and Lemma 2.2, we obtain that if $N$ is sufficiently large, then

$$
\mathbf{b}(N-e) \leq \sum_{p \leq \Theta(N)}\left(\log (A(N)+1)+\Psi(p)\left(1-\frac{\log 2}{\mathbf{h}(N)}\right)^{-1}\right)
$$


from which we deduce

$$
(\mathbf{b}(N-e)-\pi(\Theta(N)) \log (A(N)+1))\left(1-\frac{\log 2}{\mathbf{h}(N)}\right) \leq \sum_{p \leq \Theta(N)} \Psi(p) .
$$

By the assumption (2.31), we obtain $\mathbf{b}(N-e) \leq \mathbf{b}(N-d)$, which gives $e \geq d$, contradicting our assumption on $e$.

Corollary 2.4. Let $T(x)$ be a strictly increasing continuous function of the positive real variable $x$ such that the three estimates

$$
\pi(T(n)) \sim \frac{2 \mathbf{b}(n)}{\mathbf{h}(n) n\left(\log _{2} A(n)-\rho \log n\right)}
$$

for $n \rightarrow \infty$, as well as

$$
\begin{gathered}
\sum_{p \leq x} T^{-1}(p) \sim \frac{x T^{-1}(x)}{2 \log x}, \\
\frac{\lambda T(x)}{T(\lambda x)} \geq(1+o(1))
\end{gathered}
$$

for $x \rightarrow \infty$, hold, where $\lambda$ is an arbitrary parameter in $(0,1)$. Here, $T^{-1}$ is the inverse function of T. Suppose further that

$$
\log A\left(\frac{n}{2}\right) \gg \log A(n)
$$

Then

$$
\limsup _{n \rightarrow \infty} \frac{P(A(n)+1)}{T(n)} \geq 1 .
$$

Proof. It is clear that $T(N)$ tends to infinity with $N$ (see, e.g., (2.39)). We assume that the inequality (2.41) does not hold, and conclude that for some $\lambda \in(0,1)$, the inequality $P(A(N)+1)<\lambda T(N)$ holds for all $N>N_{0}$, where $N_{0}$ is some fixed positive integer.

We now use Theorem 2.3 with $d=N_{0}+1$ and $\Theta(n)=\lambda T(n)$ to get a contradiction. Recalling (2.1), we see that the hypothesis (2.37) implies that

$$
\begin{aligned}
\pi(\Theta(N)) & \ll \pi(T(N)) \ll \frac{\mathbf{b}(N)}{\mathbf{h}(N) N\left(\log _{2} A(N)-\rho \log N\right)} \\
& \ll \frac{N}{(1-\rho) \log N} \ll \frac{N}{\log N},
\end{aligned}
$$

which in turn implies that $\Theta(N) \ll N$. Thus,

$$
\pi(\Theta(N)) \log (A(N)+1) \ll \frac{N \log A(N)}{\log N} .
$$

Hypothesis (2.40) gives $\mathbf{b}(N) \gg N \log A(N)$. So, the left-hand side of the inequality $(2.31)$ (with $d=N_{0}+1$ ) is asymptotically equivalent to $\mathbf{b}(N)$. 
3064 Prime divisors of some shifted products

Note now that by the definition of $\vartheta(p)$ and our assumption, we have that $T(\vartheta(p))>p$ for all primes $p$ which divide $A(n)+1$ for some $n=1, \ldots, N$. Hence,

$$
\Delta(p) \leq N-T^{-1}(p)
$$

Using the estimate (2.38), we get

$$
\sum_{p \leq \Theta(N)} \Delta(p) \leq \sum_{p \leq \Theta(N)}\left(N-T^{-1}(p)\right) \leq N \pi(\Theta(N))-(1+o(1)) \frac{\Theta(N) T^{-1}(\Theta(N))}{2 \log \Theta(N)}
$$

Note that $T^{-1}(\Theta(N)) \geq(1+o(1)) \Theta(N)$. Indeed, by the monotonicity and continuity of $T$, this is equivalent to

$$
\Theta(N) \geq(1+o(1)) T(\lambda N)
$$

which is guaranteed by the estimate (2.39) as $N \rightarrow \infty$. Hence,

$$
\begin{aligned}
\sum_{p \leq \Theta(N)} \Delta(p) & \leq N \pi(\Theta(N))-(1+o(1)) \pi(\Theta(N)) \frac{\Theta(N)}{2} \\
& \leq N \pi(\Theta(N))\left(1-\frac{\lambda}{2}+o(1)\right)
\end{aligned}
$$

Now,

$$
\begin{aligned}
\sum_{\substack{2 \leq p \leq \Theta(N) \\
\Delta(p) \leq N / \log N}} \Psi(p) \ll \mathbf{h}(N) \log _{2} A(N) \pi(\Theta(N))\left(\frac{N}{\log N}\right) \\
=O\left(\frac{\mathbf{b}(N)}{\log N}\right)=o(\mathbf{b}(N)) .
\end{aligned}
$$

Furthermore,

$$
\begin{aligned}
\sum_{\substack{\leq \leq p \leq \Theta(N) \\
\Delta(p) \geq N / \log N}} \Psi(p) & \leq \mathbf{h}(N)\left(\log _{2} A(N)-\rho \log \left(\frac{N}{\log N}\right)\right) \sum_{p=2}^{N} \Delta(p) \\
& \leq \frac{(2-\lambda+o(1)) \mathbf{h}(N)\left(\log _{2} A(N)-\rho \log N\right) N \pi(\Theta(N))}{2} \\
& \sim \frac{\lambda(2-\lambda) \mathbf{h}(N)\left(\log _{2} A(N)-\rho \log N\right) N \pi(T(N))}{2} .
\end{aligned}
$$


Eric Levieil et al. 3065

Therefore,

$$
\sum_{\substack{2 \leq p \leq \Theta(N) \\ \Delta(p) \geq N / \log N}} \Psi(p) \sim \lambda(2-\lambda) \mathbf{b}(N) .
$$

Hence, from the estimates (2.48) and (2.50), we get

$$
\sum_{p \leq \Theta(N)} \Psi(p) \leq(1+o(1)) \lambda(2-\lambda) \mathbf{b}(N)
$$

Since $\lambda(2-\lambda)<1$ for $\lambda<1$, the inequality (2.31) is satisfied for sufficiently large $N$, which is the desired contradiction.

Corollary 2.5. Suppose that all the hypotheses of Corollary 2.4 are satisfied by the function $T(n)=\mu n$. Suppose moreover that the inequality $\eta(p) \leq \nu p$ holds on a set of primes $p$ of relative asymptotic density at least $\zeta$, where $\mu, \nu>0$ and $\zeta \geq 0$ are three constants such that $\mu \nu \geq 1-\zeta$. Then the inequality

$$
\limsup _{n \rightarrow \infty} \frac{P(A(n)+1)}{n} \geq \mu+\frac{\zeta}{v}
$$

holds.

Proof. We put

$$
\mu_{0}=\mu+\frac{\zeta}{\nu}, \quad T_{0}(n)=\mu_{0} n
$$

We note that the estimates (2.37), (2.38), and (2.39) are satisfied when the function $T(x)$ is replaced by the function $T_{0}(x)$. The estimate (2.37) gives

$$
\mathbf{b}(N) \sim \frac{\mu N^{2}}{2 \log N} \mathbf{h}(N)\left(\log _{2} A(N)-\rho \log N\right) .
$$

We assume that the estimate

$$
\limsup _{n \rightarrow \infty} \frac{P(A(n)+1)}{T_{0}(n)} \geq 1
$$

does not hold, and conclude that there exist $\lambda \in(0,1)$ such that $P(A(N)+1)<\lambda T_{0}(N)$ holds for $N>N_{0}$, where $N_{0}$ is some fixed positive integer.

Now, as in the proof of Corollary 2.4, we apply Theorem 2.3 with $d=N_{0}+1$ and sufficiently large positive integer $N$.

The argument from the beginning of the proof of Corollary 2.4 shows that if we put $\Theta(N)=\lambda T_{0}(N)$, then the left-hand side of the inequality (2.31) is equivalent to $\mathbf{b}(N)$. Furthermore, the estimates (2.48) and (2.50) show that

$$
\sum_{p \leq \Theta(N)} \Psi(p) \sim \mathbf{h}(N)\left(\log _{2} A(N)-\rho \log N\right) \sum_{p \leq \Theta(N)} \Delta(p)
$$


Thus, the inequality (2.31) takes the simpler form

$$
(1+o(1)) \frac{\mu N^{2}}{2 \log N} \geq \sum_{p \leq \Theta(N)} \Delta(p) .
$$

It remains to find an upper bound for

$$
\sum_{p \leq \Theta(N)} \Delta(p)=\sum_{p \leq \Theta(N)} \eta(p)-\sum_{p \leq \Theta(N)} \vartheta(p) .
$$

It is clear that the inequality $\eta(p) \leq N$ holds for all prime $p$ and by hypothesis, the inequality $\eta(p) \leq \nu p$ holds on a set of primes $p$ of relative asymptotic density $\zeta$. Note that when $N / \nu \leq p \leq \lambda \mu_{0} N$, only the inequality $\eta(p) \leq N$ is relevant. Thus,

$$
\begin{aligned}
\sum_{p \leq \Theta(N)} \eta(p) & \leq \sum_{N / \nu \leq p \leq \lambda \mu_{0} N} N+\sum_{p=2}^{N / \nu} \eta(p) \\
& \leq N\left(\pi\left(\lambda \mu_{0} N\right)-\pi\left(\frac{N}{\nu}\right)\right)+(1-\zeta+o(1)) N \pi\left(\frac{N}{\nu}\right)+(\zeta+o(1)) \sum_{p=2}^{N / v} \nu p \\
& \sim \frac{N^{2}}{\log N}\left(\lambda \mu_{0}-\frac{1}{\nu}\right)+\frac{N^{2}}{\log N}\left(\frac{1-\zeta}{\nu}+\frac{\zeta}{2 \nu}\right) \sim \frac{N^{2}}{\log N}\left(\lambda \mu_{0}-\frac{\zeta}{2 \nu}\right)
\end{aligned}
$$

say, where $\eta(p) \leq v p$ holds on a set of relative asymptotic density $\zeta$ of primes $p \leq N / v$ as $N \rightarrow \infty$, and $\eta(p) \leq N$ otherwise.

As in the proof of Corollary 2.4, we have $\vartheta(p)>T_{0}^{-1}(p)=p / \mu_{0}$, therefore

$$
\sum_{p \leq \Theta(N)} \vartheta(p) \geq \sum_{p=2}^{\lambda \mu_{0} N} \frac{p}{\mu_{0}}=(1+o(1)) \frac{\lambda^{2} \mu_{0} N^{2}}{2 \log N}
$$

Hence, from the above estimates, we derive

$$
\begin{aligned}
\sum_{p \leq \Theta(N)} \Delta(p) & \leq(1+o(1)) \frac{N^{2}}{2 \log N}\left(2 \lambda \mu_{0}-\frac{\zeta}{\nu}-\lambda^{2} \mu_{0}\right) \\
& =(1+o(1)) \frac{N^{2}}{2 \log N}\left(\lambda(2-\lambda) \mu_{0}-\frac{\zeta}{\nu}\right) .
\end{aligned}
$$

Since

$$
\lambda(2-\lambda) \mu_{0}-\frac{\zeta}{\nu}<\mu_{0}-\frac{\zeta}{\nu}=\mu
$$

holds for all $\lambda \in(0,1)$, we see that the inequality (2.57) also holds, and now Theorem 2.3 yields the desired contradiction.

When no arithmetic properties of the sequence of numbers $(a(n))_{n \geq 1}^{N}$ are known, we simply have to take $\eta(p)=N$ and $\rho=0$ for all primes $p$. 
We recall that for any increasing function $f$,

$$
\sum_{n=1}^{t} f(n)=\int_{1}^{t} f(s) d s+O(f(t))
$$

For example, suppose that $a(n)$ is an integer for $n=1, \ldots, N$. In this case, $a(n)=h(n)$ for all $n=1, \ldots, N$. Assume that

$$
\log a(n)=K n^{\alpha}(\log n)^{\beta}(1+o(1))
$$

holds for $n=1, \ldots, N$, where $\alpha, \beta \geq 0$ and $K>0$ are constants. Then

$$
\log A(n)=\sum_{i=1}^{n} \log a(i)=\frac{K}{\alpha+1} n^{\alpha+1}(\log n)^{\beta}(1+o(1))
$$

so

$$
\begin{aligned}
& \mathbf{b}(n)=\sum_{i=1}^{n} \log A(i)=\frac{K n^{\alpha+2}(\log n)^{\beta}}{(\alpha+1)(\alpha+2)}(1+o(1)), \\
& T(n) \sim \frac{2 \mathbf{b}(n) \log n}{\mathbf{h}(n) n \log _{2} A(n)} \sim \frac{2 n}{(\alpha+1)^{2}(\alpha+2)} .
\end{aligned}
$$

\section{Applications}

In this section, we give applications of our results to various sequences which arise in combinatorics and number theory. Typically, but not always, the growth conditions are readily available, while for several of them lower bounds on $\rho$ have been given in [1].

3.1. Number-theoretic functions. Interesting results are obtained when the sequence $a(n)$ has integer values and both $\log a(n)$ and $\log A(n)$ have mean values which are considerably smaller (by an order of magnitude) than their maximal values for $n=1, \ldots, N$. In this section, we look at the instance in which $a(n)$ is one of the classical arithmetic functions of $n$.

Lemma 3.1. The following estimates hold:

$$
\begin{aligned}
& \sum_{n \leq N} \log \omega(n)=(1+o(1)) N \log _{3} N \\
& \sum_{n \leq N} \log \Omega(n)=(1+o(1)) N \log _{3} N \\
& \sum_{n \leq N} \log \tau(n)=(\log 2+o(1)) N \log _{2} N
\end{aligned}
$$


3068 Prime divisors of some shifted products

Proof. Let

$$
\begin{aligned}
& \mathscr{A}_{1}=\left\{n \leq N:\left|\omega(n)-\log _{2} N\right| \leq\left(\log _{2} N\right)^{2 / 3}\right\} ; \\
& \mathscr{A}_{2}=\left\{n \leq N:\left|\omega(n)-\log _{2} N\right|>\left(\log _{2} N\right)^{2 / 3}, \omega(n) \leq\left(\log _{2} N\right)^{3 / 2}\right\} ; \\
& \mathscr{A}_{3}=\left\{n \leq N: \omega(n) \geq\left(\log _{2} N\right)^{3 / 2}\right\} .
\end{aligned}
$$

By the Turán-Kubilius estimate (see, e.g., [7]),

$$
\sum_{n \leq N}\left(\omega(n)-\log _{2} N\right)^{2}=O\left(N \log _{2} N\right)
$$

we get that $\# \mathscr{A}_{2} \ll N /\left(\log _{2} N\right)^{1 / 3}$ and $\# \mathscr{A}_{3} \ll N /\left(\log _{2} N\right)^{2}$. Since we also have that $\log \omega(n) \ll$ $\log _{3} N$ for $n \in \mathscr{A}_{2}$ and $\log \omega(n) \ll \log _{2} N$ for $n \in \mathscr{A}_{3}$, we get that

$$
\sum_{n \in \mathcal{A}_{2} \cup \mathscr{A}_{3}} \log \omega(n) \ll \frac{N \log _{3} N}{(\log N)^{1 / 3}} .
$$

Since for $n \in \mathscr{A}_{1}$, we have

$$
\log \omega(n)=\log _{3} N+\log \left(\frac{\omega(n)}{\log _{2} N}\right)=\log _{3} N+O\left(\frac{1}{(\log N)^{1 / 3}}\right),
$$

we get the desired result for $\omega(n)$. The bound for $\Omega(n)$ follows from entirely similar arguments.

We now denote by $p^{\alpha} \| n$ the exact divisibility by $p^{\alpha}$ (meaning that the $p$-adic order of $n$ is $\alpha$ ). Then

$$
\begin{aligned}
\sum_{n \leq N} \log \tau(n) & =\sum_{n \leq N} \sum_{p^{\alpha} \| n} \log (\alpha+1)=\sum_{p^{\alpha} \leq N} \log (\alpha+1) \sum_{\substack{n \leq N \\
p^{\alpha} \| n}} 1 \\
& =\sum_{p^{\alpha} \leq N} \log (\alpha+1)\left(\frac{N}{p^{\alpha}}-\frac{N}{p^{\alpha+1}}+O(1)\right) \\
& =\sum_{p^{\alpha} \leq N} \log (\alpha+1)\left(\frac{N(p-1)}{p^{\alpha+1}}+O(1)\right) .
\end{aligned}
$$

Since $\alpha \leq \log N / \log 2 \leq 2 \log N$ and there are $O(N / \log N)$ prime powers $p^{\alpha} \leq N$, we derive

$$
\begin{aligned}
\sum_{n \leq N} \log \tau(n) & =N \sum_{p^{\alpha} \leq N} \frac{(p-1) \log (\alpha+1)}{p^{\alpha+1}}+O(N) \\
& =N \log 2 \sum_{p \leq N} \frac{(p-1)}{p^{2}}+N \sum_{\substack{\alpha \geq 2 \\
p^{\alpha} \leq N}} \frac{(p-1) \log (\alpha+1)}{p^{\alpha+1}}+O(N) .
\end{aligned}
$$


Eric Levieil et al. 3069

By the Mertens formula,

$$
\sum_{p \leq N} \frac{(p-1)}{p^{2}}=\sum_{p \leq N} \frac{1}{p}+O(1)=\log _{2} N+O(1) .
$$

We also have

$$
\begin{aligned}
\sum_{\substack{\alpha \geq 2 \\
p^{\alpha} \leq N}} \frac{(p-1) \log (\alpha+1)}{p^{\alpha+1}} & \ll N \sum_{\alpha \leq 2 \log N} \log (\alpha+1) \sum_{m=2}^{\infty} \frac{1}{m^{\alpha}} \\
& \leq \sum_{\alpha \leq 2 \log N} \log (\alpha+1)\left(2^{-\alpha}+\int_{2}^{\infty} z^{-\alpha} d z\right) \\
& \ll N \sum_{\alpha \leq 2 \log N} \frac{\log (\alpha+1)}{2^{\alpha}} \ll N,
\end{aligned}
$$

which finishes the proof.

We are now ready to prove the following result.

TheOREM 3.2. The following estimates hold:

$$
\begin{gathered}
\limsup _{n \rightarrow \infty} \frac{P\left(\prod_{i=1}^{n} \varphi(i)+1\right)}{n} \geq 1 ; \\
\limsup _{n \rightarrow \infty} \frac{P\left(\prod_{i=1}^{n} \sigma(i)+1\right)}{n} \geq 1 ; \\
\limsup _{n \rightarrow \infty} \frac{P\left(\prod_{i=1}^{n} \omega(i)+1\right) \log _{2} n}{n \log _{3} n} \geq 1 ; \\
\limsup _{n \rightarrow \infty} \frac{P\left(\prod_{i=1}^{n} \Omega(i)+1\right) \log _{2} n}{n \log _{3} n} \geq 1 ; \\
\limsup _{n \rightarrow \infty} \frac{P\left(\prod_{i=1}^{n} \tau(i)+1\right) \log _{n}}{n\left(\log _{2} n\right)^{2}} \geq 1 .
\end{gathered}
$$

Proof. Since $n / \log _{2} n \ll \varphi(n) \leq n$ and $n \leq \sigma(n) \ll n \log _{2} n$, it follows that if $a(n)=\varphi(n)$ or $\sigma(n)$, then the estimate (2.64) holds with $\alpha=0$. The estimates for the products of $\varphi(i)$ and $\sigma(i)$ now follow from Corollary 2.4.

When $a(i)=\omega\left(m_{i}\right)$, where $m_{i}=i+O(i / \log i)$ is the $i$ th composite number (we need to skip prime numbers because of the condition $a(i)>1$ used in Section 2), we apply Lemma 3.1 to get that

$$
\log A(t)=\sum_{i \leq t} \log \omega\left(m_{i}\right) \sim t \log _{3} t
$$


therefore

$$
\mathbf{b}(n)=\sum_{i=1}^{n} \log A(i)=\int_{1}^{n} \log A(t) d t \sim \frac{1}{2} n^{2} \log _{3} n .
$$

We take $\eta(p)=N, \rho=0$, and use the fact that

$$
\log \omega\left(m_{i}\right) \leq(1+o(1)) \log _{2} N, \quad 1 \leq i \leq N,
$$

to get that

$$
T(n) \sim \frac{n \log _{3}(n)}{\log _{2} n},
$$

which gives the inequality for the products of $\omega(i)$.

The inequality for the products of $\Omega(i)$ is entirely similar, and the last inequality follows again by Lemma 3.1 together with the inequality

$$
\log \tau(n) \leq(\log 2+o(1)) \frac{\log N}{\log _{2} N}, \quad 1 \leq n \leq N,
$$

which finishes the proof.

We remark that we could have taken, say, $\eta(p)=p^{2}$ when $a(n)=\varphi(n)$ (because $p \mid$ $\left.\varphi\left(p^{2}\right)\right), \eta(p)=2^{p-1}$ when $a(n)=\Omega(n)$, and

$$
\eta(p)=\prod_{\substack{q \leq p \\ q \text { prime }}} q
$$

when $a(n)=\omega(n)$ or $a(n)=\tau(n)$, but such choices do not seem to lead to any substantial improvements. The widely believed conjecture asserting that the first prime number $q \equiv 1(\bmod p)$ satisfies $q \ll p^{1+\varepsilon}$ would give that $\eta(p)=O\left(p^{1+\varepsilon}\right)$ for $a(n)=\varphi(n)$ (because $p \mid \varphi(q)$ ), but otherwise would not lead to any improvements of the inequality of Theorem 3.2 for the Euler function either. Similar remarks apply to the sum of divisors function.

3.2. Polynomials. Let $a(n)=|g(n)|$, where $g(X) \in \mathbb{Q}[X]$ is a nonconstant polynomial with $|g(n)| \geq 1$ for $n \geq 1$.

In this case, the estimate (2.64) holds with $\alpha=0, \beta=1$, and $K=\operatorname{deg} g$. Thus,

$$
\mathbf{b}(n)=\frac{d}{2} n^{2} \log n(1+o(1)) .
$$

We can take $\rho(p)=1 / 3$ (see $[1])$, so $T(n) \sim 3 n / 2$.

Moreover, the set of prime numbers $p$ such that the polynomial $g(X)$ has a zero modulo $p$ has a relative density $\delta>0$ (in the set of all prime numbers), by the Chebotarev density theorem. For such primes, we can take $n(p)$ to be the minimum between $N$ and the smallest zero modulo $p$ of $a(n)$, which is at most $p$. 
So, using Corollary 2.5 with $\mu=3 / 2, \nu=1, \zeta=\delta$, we obtain the following.

Theorem 3.3. Let $g(X) \in \mathbb{Q}[X]$ be a nonconstant integer-valued polynomial of $n$ with $|g(n)| \geq 1$ for $n \geq 1$. Let $\delta$ be the density of the subset of primes $p$ such that the congruence $g(n) \equiv 0(\bmod p)$ has an integer solution $n$. Then the inequality

$$
\limsup _{n \rightarrow \infty} \frac{P\left(\prod_{i=1}^{n}|g(i)|+1\right)}{n} \geq \frac{3}{2}+\delta
$$

holds.

Note that, by the Chebotarev density theorem, we know in fact that $\delta \geq 1 /(\operatorname{deg} g)$ !, and that it can be explicitly computed. When $g(X)=X$, we have $\delta=1$, and we recover the main result of [3].

3.3. Motzkin, Schröder, and Bell numbers. Using Corollary 2.4, the above result allows us to write down nontrivial lower bounds for various numbers arising from enumerative combinatorics. Recall that Motzkin numbers $m_{n}$ count, for a positive integer $n$, the number of lattice paths starting at $(0,0)$, ending at $(0, n)$, which use line steps parallel to $(1,0)$ (level step), $(1,1)$ (up step), or $(1,-1)$ (down step), and which never pass below the $x$-axis. The Schröder numbers $s_{n}$ are defined similarly except that the ending point of the paths is $(2 n, 0)$, and the level step is $(2,0)$. Finally, the Bell numbers $B_{n}$ count the number of ways of partitioning a set with $n$ elements into disjoint nonempty subsets. Since $a(n)$ satisfies (2.64) with $\alpha=1$ and some $\beta \geq 0$ whenever $a(n)$ is one of $m_{n}, s_{n}$, or $B_{n}$, we have obtained the following result.

Theorem 3.4. The inequality

$$
\limsup _{n \rightarrow \infty} \frac{P(A(n)+1)}{n} \geq \frac{1}{6}
$$

holds whenever $(a(n))_{n=1}$ is the sequence of the Motzkin numbers, or Schröder numbers, or Bell numbers.

Lower bounds of the form $P(A(N)) \gg \log N \log _{2} N$ have been obtained in [2] when $a(i)=m_{i}$ for $i=1, \ldots, N$ (and the method there works for $a(i)=s_{i}$ for $i=1, \ldots, N$, as well), and of the form $P(A(N)) \gg \log N$ when $a(i)=B_{i}$ for $i=1, \ldots, N$, in [5].

3.4. Middle binomial coefficients and Catalan numbers. Here, we take

$$
b(n)=\left(\begin{array}{c}
2 n \\
n
\end{array}\right) \quad \text { or } \quad c(n)=\frac{1}{n+1}\left(\begin{array}{c}
2 n \\
n
\end{array}\right),
$$

$n=1, \ldots, N$, that is, $b(n)$ is the $n$th middle binomial coefficient and $c(n)$ the $n$th Catalan number, respectively. In this case, using the Stirling formula, it is easily seen that both sequences $a(n)=b(n)$ and $a(n)=c(n)$ satisfy formula (2.64) with $\alpha=1, \beta=0$, and $K=$ $2 \log 2$. We can take $\rho(p)=1 / 5$ (see [1]), so $T(n) \sim 5 n / 4$. 
3072 Prime divisors of some shifted products

Obviously,

$$
p \mid\left(\begin{array}{c}
p+1 \\
p+1 / 2
\end{array}\right)
$$

so we can take $\eta(p)=(p-1) / 2$.

We now take $\mu=5 / 4, \nu=1 / 2, \zeta=1$ in Corollary 2.5, to get the following.

Theorem 3.5. The inequality

$$
\limsup _{n \rightarrow \infty} \frac{P\left(\prod_{i=1}^{n} a(i)+1\right)}{n} \geq \frac{13}{4}
$$

holds where either $a(i)=b(i)$ is the ith middle binomial coefficient or $a(i)=c(i)$ is the ith Catalan numbers.

The methods of this section apply to more general sequences such as hypergeometric, that is, of the form $a(n)=\prod_{i=1}^{n} f(i)$, where $f \in \mathbb{Q}[X]$ is a nonzero integer-valued polynomial.

3.5. $q$-factorials. We let $q>1$ be an integer and set $a(n)=q^{n}-1$ for $n=1, \ldots, N$. The estimate (2.64) holds with $\alpha=1, \beta=0$, and $K=\log q$. We have also $\eta(p) \leq p-1$, by the Fermat's little theorem, for all but finitely many primes $p$ (i.e., except for the prime factors of $q$ ). Moreover, $\rho(p) \geq 1 / 3$ for all primes $p$ (see [1]). Therefore, we can take $\mu=1 / 4, \nu=1, \zeta=1$. Using Corollary 2.5, we obtain the following.

Theorem 3.6.

$$
\limsup _{n \rightarrow \infty} \frac{P\left(\prod_{i=1}^{n}\left(q^{i}-1\right)+1\right)}{n} \geq \frac{5}{4}
$$

The results of this section remain also valid if the sequence $\left(q^{n}-1\right)_{n=1}^{N}$ is replaced by the first $N$ terms of some other Lucas sequence, like the Fibonacci sequence, for example.

\section{Acknowledgments}

We thank the anonymous referees for suggestions that greatly improved the quality of this paper. During the preparation of this paper, the second author was supported in part by Grants SEP-CONACYT 37259-E and 37260-E, and the third author was supported in part by ARC Grant DP0211459.

\section{References}

[1] E. Levieil, F. Luca, and I. E. Shparlinski, Bounding the number of solutions of congruences, Bol. Soc. Mat. Mexicana 11 (2005), no. 2.

[2] F. Luca, Prime factors of Motzkin numbers, to appear in Ars Combinatoria.

[3] F. Luca and I. E. Shparlinski, Prime divisors of shifted factorials, Bull. London Math. Soc. 37 (2005), no. 6, 809-817.

[4] On the largest prime factor of $n !+2^{n}-1$, to appear in J. Théor. Nombres Bordeaux. 
[5] I. E. Shparlinski, The number of different prime divisors of recurrent sequences, Mat. Zametki 42 (1987), no. 4, 494-507, 622 (Russian).

[6] C. L. Stewart, On the greatest and least prime factors of $n !+1$. II, Publ. Math. Debrecen 65 (2004), no. 3-4, 461-480.

[7] P. Turán, On a theorem of Hardy and Ramanujan, J. London Math. Soc. 9 (1934), 274-276.

Eric Levieil: Département d’Informatique, École Normale Supérieure 45, rue d’Ulm, 75230 Paris Cedex 05, France

E-mail address: eric.levieil@ens.fr

Florian Luca: Instituto de Matemáticas, Universidad Nacional Autónoma de México, 58089 Morelia, Michoacán, Mexico

E-mail address: fluca@matmor.unam.mx

Igor E. Shparlinski: Department of Computing, Division of Information and Communication Sciences, Macquarie University, Sydney, NSW 2109, Australia

E-mail address: igor@ics.mq.edu.au 


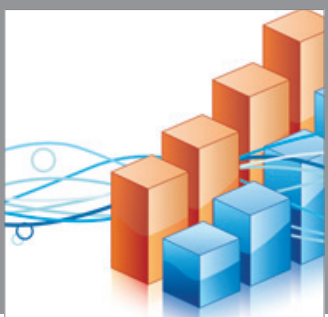

Advances in

Operations Research

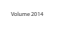

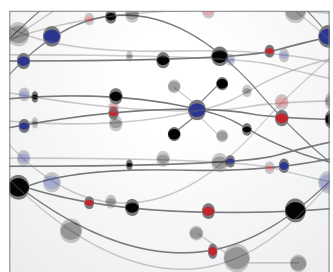

\section{The Scientific} World Journal
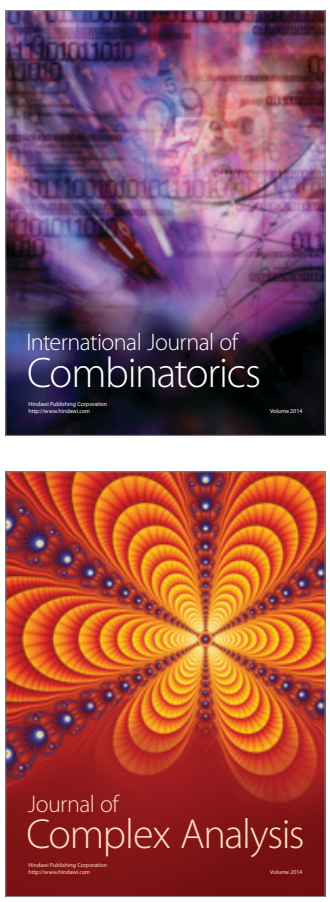

International Journal of

Mathematics and

Mathematical

Sciences
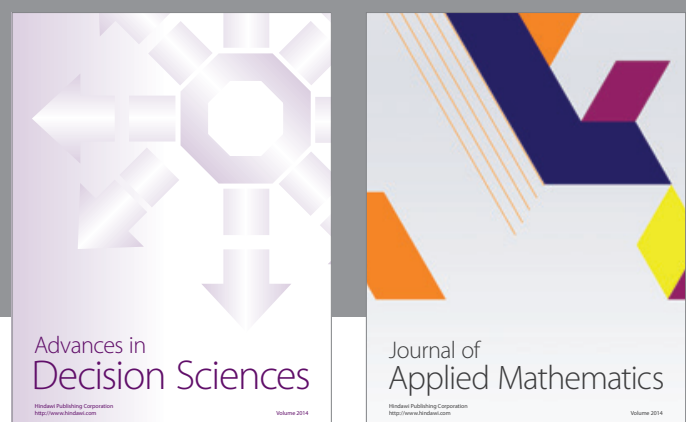

Journal of

Applied Mathematics
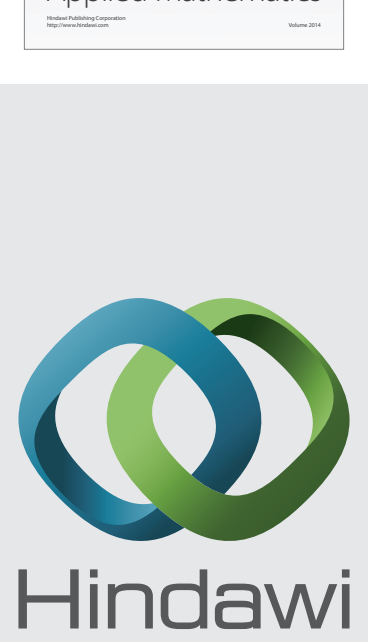

Submit your manuscripts at http://www.hindawi.com
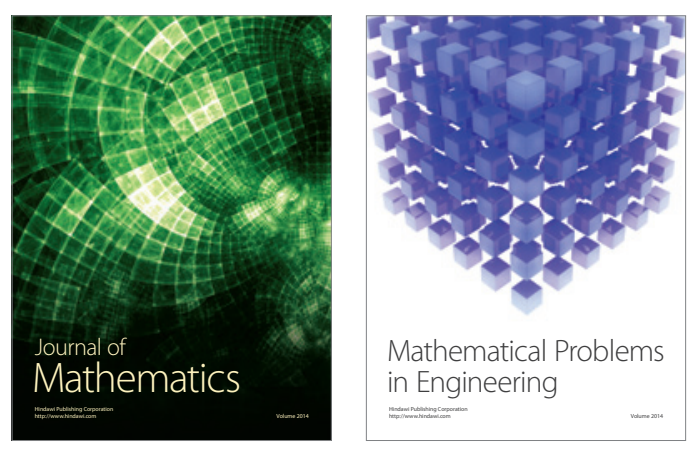

Mathematical Problems in Engineering
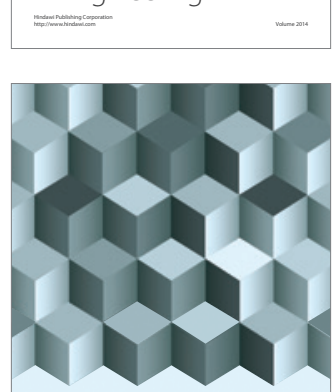

Journal of

Function Spaces
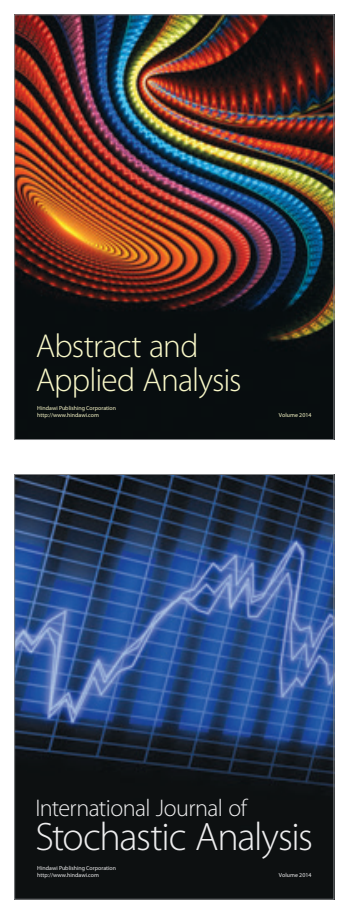

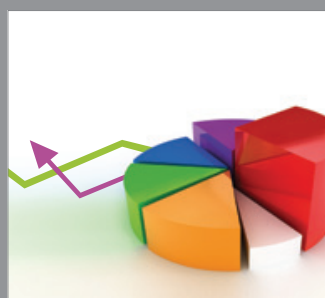

ournal of

Probability and Statistics

Promensencen
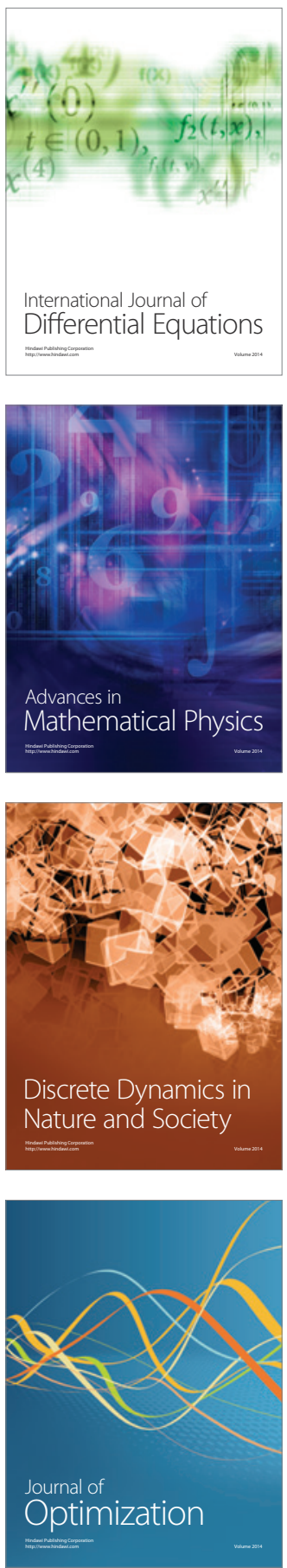